\title{
Variabilidad oceánica y cambios de nivel del mar alrededor de la península ibérica, Baleares y Canarias

\section{Jordi Salat' ${ }^{1}$, Alicia Lavín ${ }^{4}$, César González-Pola ${ }^{2}$, Pedro Vélez-Belchí ${ }^{2}$, Ricardo Sánchez ${ }^{2}$, Manolo Vargas-Yáñez ${ }^{2}$, Jesús García-Lafuente ${ }^{3}$, Marta Marcos $^{4}$, Damià Gomis ${ }^{4}$}

\author{
${ }^{1}$ Institut de Ciències del Mar (CSIC), España \\ ${ }^{2}$ Instituto Español de Oceanografía (IEO), España \\ ${ }^{3}$ Universidad de Málaga, España \\ ${ }^{4}$ Institut Mediterrani d'Estudis Avançats (IMEDEA: Universitat de les Illes Balears - CSIC), España
}

\begin{abstract}
Introducción
El océano, con una capacidad de almacenamiento de calor casi 1200 veces superior a la de la atmósfera, es el principal componente, en términos de balance de energía, del sistema climático de la Tierra. Uno de los objetivos de CLIVAR es obtener la máxima información posible sobre la variabilidad oceánica, incluyendo cambios en el nivel del mar, como consecuencia del almacenamiento de calor en el océano. Esta información es utilizada para mejorar la comprensión de la variabilidad climática a escala interanual, para detectar cambios en el sistema climático y los flujos de energía, y para ajustar y optimizar modelos que, a su vez, permitan mejorar tanto las predicciones de cambio climático tanto a escala de décadas como las proyecciones a más largo plazo. En un contexto regional, las aguas alrededor de la península ibérica (incluidas las islas Baleares) y alrededor de las islas Canarias están afectadas principalmente por la variabilidad del Atlántico nororiental y del Mediterráneo occidental, incluyendo la comunicación entre ambas cuencas. En la anterior evaluación de CLIVAR-España (2010), se analizaron y discutieron los resultados básicos sobre variabilidad oceánica y cambios del nivel del mar en la región, basados en observaciones in situ a largo plazo e imágenes de satélite. En aquella evaluación se describió la evolución de las características del agua (tendencias y escalas de variabilidad de temperatura, salinidad, contenido de calor y de nivel del mar) desde las primeras observaciones hasta el primer decenio de este siglo.
\end{abstract}

Como principales resultados de aquel informe cabe destacar, en primer lugar, que las aguas superficiales en todo el margen atlántico de la península ibérica presentaban alternancia entre periodos fríos y cálidos desde 1854. El último periodo cálido dio comienzo en 1974 . Se describió una tendencia al calentamiento claramente detectada en el golfo de Vizcaya durante la década de los noventa, que se extendía a toda la capa superior del océano hasta $1000 \mathrm{~m}$ de profundidad. Las aguas oceánicas del Atlántico subtropical alrededor de las islas Canarias presentaban una tendencia al calentamiento y un incremento de salinidad a profundidades de entre 600 y $1800 \mathrm{~m}$, probablemente asociados a variaciones en la intensidad de los vientos dominantes a través de su impacto en los desplazamientos verticales de las masas de agua. También se constató un reforzamiento del afloramiento del noroeste de África, o por lo menos un debilitamiento de la tendencia al calentamiento de las aguas superficiales de la región tropical del Atlántico que se venía observando desde 1967. En el Mediterráneo occidental se observó un aumento de temperatura y salinidad de las aguas profundas, así como de la salinidad de las aguas intermedias, desde mediados del siglo XX. Sin embargo, según el mencionado informe, las estimaciones de flujos de calor en la región ibérica no presentaban ninguna tendencia significativa, tan solo algunas alternancias entre periodos de mayor o menor transferencia de calor del mar a la atmósfera (por ejemplo, de 1958 a 1975, y entre 1975 y 2001, respectivamente). En ese contexto, se destacaron por su relevancia los elevados flujos de calor hacia la atmósfera, observados durante el invierno de 2004/2005, que provocaron la formación de grandes capas de mezcla vertical en una extensa región del Mediterráneo noroccidental y la producción de un volumen excepcional de agua densa, sin precedentes en la región.

Con respecto a la variabilidad a largo plazo del nivel medio del mar, las observaciones referidas en el informe indicaban tendencias crecientes, aunque de diversas magnitudes. Como resultado destacable, cabe indicar que desde los años sesenta hasta los noventa, no se observó ningún aumento del nivel del mar en el Mediterráneo occidental debido al forzamiento negativo causado por el aumento de la presión atmosférica. Sin embargo, desde entonces el nivel del mar ha vuelto a aumentar siguiendo la tendencia positiva global. Asimismo, en lo referente a los valores extremos del nivel del mar, se constató que tanto su evolución a largo plazo como su variabilidad interanual eran consistentes con el comportamiento del nivel medio, a pesar de la diversidad de procesos que causan los extremos, tales como la interacción entre marea y tormentas. Ello supone una comprobación indirecta de que durante las últimas décadas del siglo XX no habría habido cambios importantes, ni en frecuencia ni en intensidad, en las perturbaciones atmosféricas que afectan a las costas de la península ibérica. 

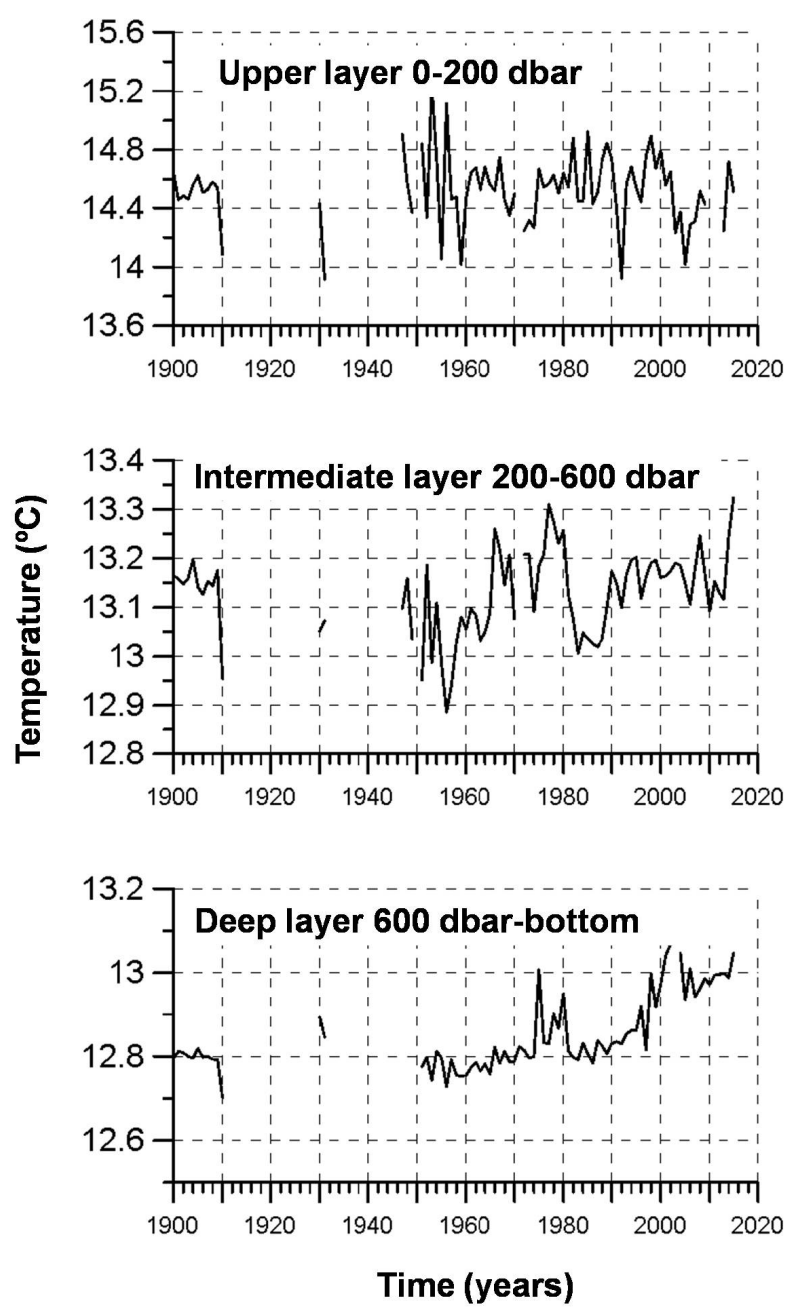

Figura 1. Series de temperatura en tres capas, de 1900 a 2015, promediada anualmente en toda la región española del Mediterráneo occidental. Adaptado de Vargas-Yáñez et al. (2017).

En este trabajo se presentan nuevos resultados, obtenidos de las observaciones de los últimos diez años, la mayoría basados en nuevos conjuntos de datos. Su objetivo es actualizar y revisar los resultados anteriores, así como tener una perspectiva detallada de los cambios interanuales observados durante la última década. La mayoría de los datos provienen de los mismos programas de seguimiento que figuraban en el informe anterior: la red de medidores del nivel del mar, secciones de CTD y estaciones fijas repetidas estacionalmente, y anclajes desplegados en puntos clave de las cuencas marinas y en el estrecho de Gibraltar. Para medir la temperatura superficial y el nivel del mar se ha usado, además, la teledetección desde satélites. Más allá de los datos provenientes de seguimientos sistemáticos, los datos de campañas oceanográficas y de algunos anclajes ocasionales también han contribuido a los resultados analizados en este artículo. También datos de planeadores marinos («gliders»), boyas de deriva instrumentadas y, muy especialmente, de perfiladores ARGO, que hoy en día se han convertido en esenciales (no se utilizaron en el informe anterior porque su expansión empezó hace tan solo unos pocos años). Por contraposición, cabe señalar que observaciones reales de los flujos de calor, especialmente de los intercambios de calor latente, aunque son cruciales para los fines de CLIVAR aún no están disponibles a día de hoy; por lo tanto, los resultados de los flujos de calor todavía se basan principalmente en reanálisis o en estimaciones indirectas.

\section{Resultados \\ Aguas alrededor de la península ibérica, incluyendo las islas Baleares}

Las series temporales analizadas muestran una marcada variabilidad, con cambios bruscos en la mayor parte de ellas, debido a la respuesta no lineal del sistema océano-atmósfera. Ello no entra en contradicción con la existencia de tendencias de las propiedades hidrográficas sostenidas a largo plazo, sino que revela una heterogeneidad regional en las respuestas. La característica más destacada de este último periodo es todavía fruto del anómalo invierno 2004/2005, ya mencionado en el informe anterior. Ahora, con algo más de perspectiva, se confirma como el invierno más frío y uno de los más secos de los últimos 60 años en el suroeste de Europa y la zona templada del Atlántico nororiental (Häkkinen et al., 2015). Las condiciones atmosféricas en el invierno de 2005 afectaron notablemente a la capa superficial de casi todas las aguas alrededor de la península ibérica (Levitus et al., 2012). La excepcional pérdida de calor en áreas extensas provocó un aumento del espesor de la capa de mezcla hasta profundidades sin precedentes, tanto en el Atlántico NE (Somavilla et al., 2009) como en la cuenca mediterránea occidental, donde el volumen de agua profunda formada aquel año fue muy superior a la media (Ulses et al., 2008; CIESM, 2009). Los principales cambios asociados con este episodio se han observado tanto en los registros de temperatura y salinidad de superficie (Goikoetxea et al., 2009; González et al., 2013) como en las características de las masas de agua formadas en la región, concretamente: el agua central del Atlántico nororiental (en inglés, ENACW; Prieto et al., 2015) y el agua profunda de la cuenca mediterránea occidental (en inglés, WMDW; López-Jurado et al., 2005; Font et al., 2007; Schroeder et al., 2016).

Los cambios descritos también tuvieron un impacto directo sobre el contenido de calor de los océanos en toda la región, cuya consecuencia más importante fue una redistribución del calor hacia capas más profundas (a 800-1000 m en el Atlántico NE). Como consecuencia, también se detectaron cambios en los patrones de circulación debidos a la alteración de la estructura de los campos de densidad del Atlántico NE (Somavilla et al., 2016). En el Mediterráneo, la formación masiva de nueva WMDW en 2005 tuvo un impacto directo sobre la estructura de las capas más profundas, hasta tal punto que el evento ha sido denominado como el Transitorio del Mediterráneo Occidental. Es decir, la WMDW recién formada provocó un desplazamiento hacia arriba del agua profunda residente y, por lo tanto, una disminución de temperatura y salinidad en las aguas intermedias (Zunino et al., 2012) parecido a lo ocurrido en el Mediterráneo oriental a finales de los años ochenta (Theocharis et al., 2002). El impacto de la Formación de Aguas Densas Profundas (FADP) en 2004/05 sobre la estructura termohalina del Mediterráneo occidental tiene algunos precedentes, aunque 
con mucha menor intensidad (Lacombe et al., 1985), y podría plantearse como un fenómeno recurrente (Puig et al., 2013). La disminución de temperatura y salinidad en las capas intermedias post-FADP ha influido en particular sobre el flujo de salida del agua mediterránea (en inglés, MOW) observado en Gibraltar (García-Lafuente et al., 2007). La huella hidrográfica de la MOW, sin embargo, resulta difícil de seguir a lo largo de la propagación de la vena de agua mediterránea (MW, en inglés) en el Atlántico. En particular, tal como se menciona más abajo, las variaciones en las propiedades del núcleo de la MW observadas en el noroeste de la península ibérica a lo largo de los últimos 15 años han sido más bien interpretadas como una respuesta regional advectiva vinculada a la expansión y contracción de los giros subpolar y subtropical (Prieto et al., 2015). No obstante, los impactos directos e indirectos de la anomalía del invierno 2005 produjeron un cambio en la mayor parte de los registros por encima de $\sim 1000 \mathrm{~m}$ en una amplia zona del Atlántico norte. Como consecuencia relevante cabe señalar la contribución del océano Atlántico nororiental al denominado hiato del calentamiento global, a través del calor e incremento de salinidad, adquiridos progresivamente en las capas superiores del océano y transferidos rápidamente a capas más profundas (Sullivan, 2016). Según Somavilla et al. (2016), «patrones atmosféricos anómalos como los subyacentes a este cambio [2005] no son exclusivos de la última década, aunque estos puedan haberse visto acentuados debido al calentamiento global». Ello sugiere que pueden esperarse nuevos cambios relevantes en las series temporales climáticas, pero que difícilmente podrán ser anticipados.

Además del notable evento de 2004/2005, y sin una relación aparente, se pueden mencionar otros resultados relevantes:
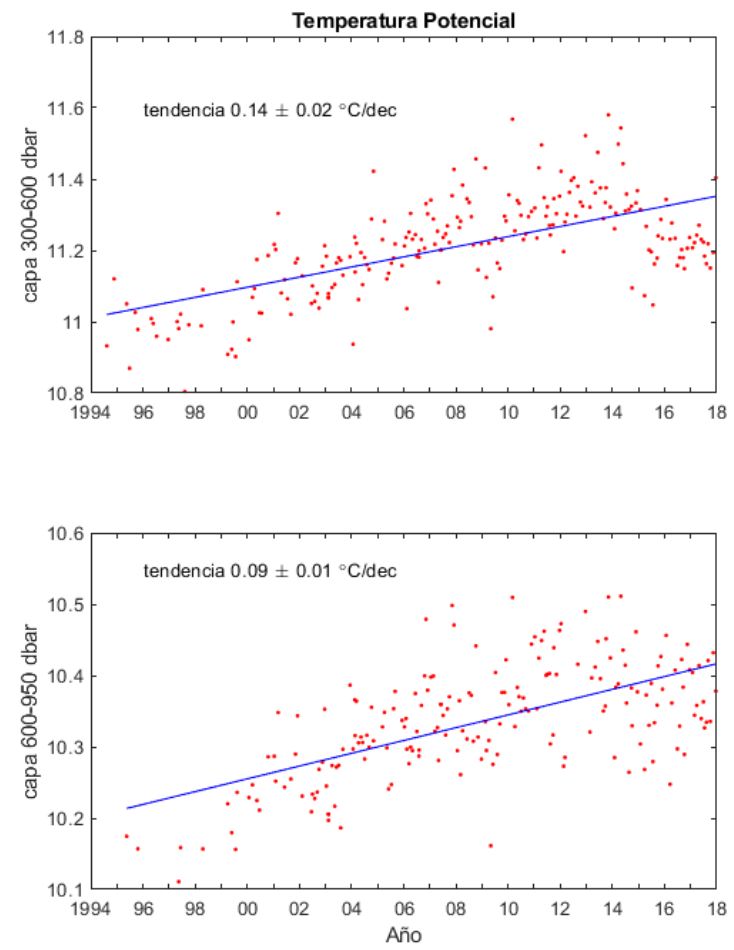

1. En 2014, la capa superior de la ENACW mostró una marcada disminución de salinidad (hasta $400 \mathrm{~m}$ ) por primera vez en casi una década (Larsen et al., 2016).

2. Se han detectado cambios significativos en la zona de transición entre la MW y el agua del mar del Labrador (LSW, en inglés), concretamente, el paso de una bolsa de agua de muy baja temperatura y salinidad de la LSW entre 2008 y 2010 (Prieto et al., 2015). Las oscilaciones en la temperatura y salinidad de esas aguas intermedias parecen estar relacionadas con la expansión/contracción del giro subpolar y la varibilidad interanual en la formación de las masas de agua en origen (Larsen et al., 2016).

3. En los años 2006, 2009, 2010, 2012 y 2013 hubo episodios claros de FADP en el Mediterráneo noroccidental (Durrieu de Madron et al., 2013). No obstante, en los tres últimos años (de 2015 a 2017) no se ha vuelto a registrar ningún episodio significativo de FADP (Durrieu de Madron et al., 2017).

4. La salinidad de la WMDW formada con posterioridad a 2005 ha ido aumentando progresivamente, especialmente en años en los que se ha formado exclusivamente por procesos de convección en mar abierto (Borghini et al., 2014).

5. Durante el episodio de FADP de 2010 se formó también una gran cantidad de agua intermedia en una zona mucho más amplia de lo habitual (Vargas-Yáñez et al., 2012).

La reciente evolución de las aguas del Mediterráneo también se ha documentado en Sammartino et al. (2014) y
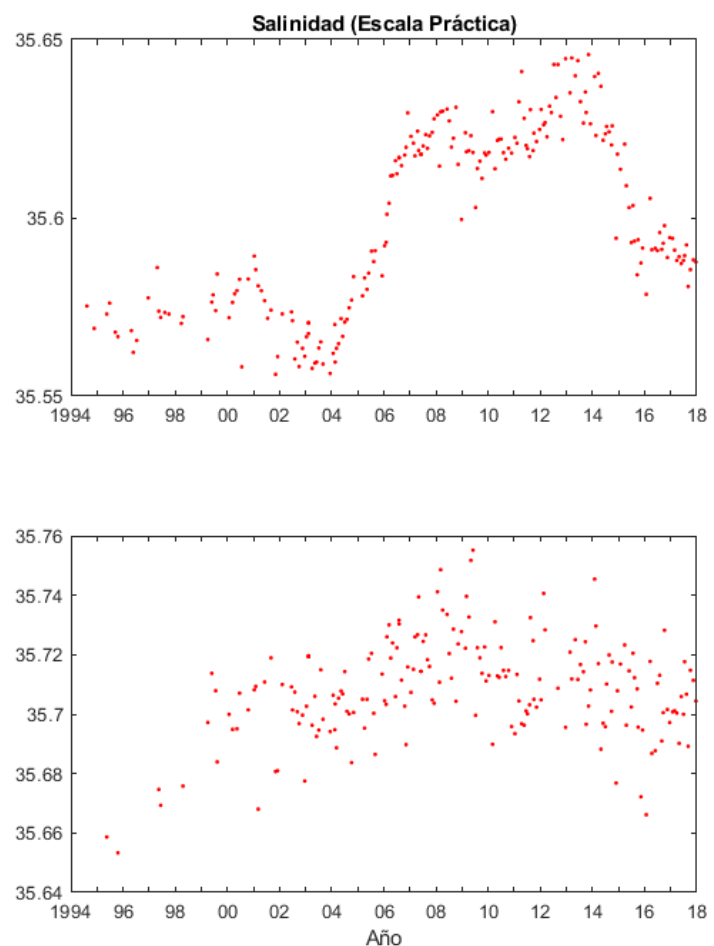

Figura 2. Series de temperatura y salinidad en niveles intermedios (300 a $1000 \mathrm{~m}$ ), obtenidas entre 1990 y 2016 en una estación de aguas abiertas situada frente a Santander (golfo de Vizcaya, $43^{\circ} 48^{\prime} \mathrm{N}, 03^{\circ} 47^{\prime} \mathrm{W} ; 2400 \mathrm{~m}$ ). 
Naranjo et al. $(2015,2017)$ en la zona del estrecho de Gibraltar.

El caudal de salida del agua del Mediterráneo en el umbral de Espartel, mostró una leve tendencia a la baja, no significativa, desde 2004 (Sammartino et al., 2014), con un ciclo estacional, con valores máximos en marzo y abril, que es cuando la interfaz se encuentra en su nivel más superficial. La salinidad, y particularmente la temperatura, de la MOW fueron bastante sensibles a este ciclo estacional, así como a la intensidad de los episodios de DWF en el Mediterráneo occidental. Desde 2004 a 2013 se detectó una tendencia ligeramente positiva tanto en temperatura (significativa al nivel de confianza del $95 \%$ ) como en salinidad (no significativa a este nivel), tendencia que aumentó en un factor de casi 4 a partir del año 2013. Esta notable aceleración del aumento en salinidad ha sido tentativamente relacionada con la WMT, a saber por el final del drenado de la WMDW previa a 2005, que había sido desplazada hacia arriba por la WMT (Naranjo et al., 2017). En la parte oriental del golfo de Cádiz, desde 1996, la capa superior de la columna de agua, hasta $150 \mathrm{~m}$, presentó un enfriamiento y disminución de salinidad y, por el contrario, en los niveles de la MOW (entre 350 y $550 \mathrm{~m}$ de profundidad), las aguas se fueron calentado y su salinidad fue en aumento (Informe de 2017 sobre Clima Oceánico (IROC) del Grupo de Trabajo sobre hidrografía oceánica (WGOH) del Consejo Internacional para la Exploración del Mar(ICES), en prensa). Estos cambios, no obstante, no han tenido ninguna influencia estadísticamente significativa en la estratificación de la columna de agua.

\section{Aguas del Atlántico subtropical alrededor de las islas Canarias}

En 2011 se repitió la sección hidrográfica transatlántica a lo largo del paralelo $24^{\circ} 30^{\prime} \mathrm{N}$, muestreada anteriormente en 1992. La comparación de los resultados entre ambas secciones muestra que ha habido un aumento en el transporte hacia el sur de la capa superior de agua profunda del Atlántico Norte (NADW, en inglés), compensado por una disminución del transporte de la capa inferior de la NADW. Asimismo, se infiere un hundimiento del límite superior del agua de fondo antártica (AABW, en inglés) desde 1992 hasta 2011. En estos casi 20 años también se observa que el flujo hacia el norte del agua intermedia antártica (AAIW, en inglés) ha disminuido considerablemente. En conjunto, pues, el transporte total de calor a través del paralelo $24^{\circ} 30^{\prime} \mathrm{N}$ no habría variado significativamente entre 1992 y 2011 (Vélez-Belchí et al., 2010; Hernández-Guerra et al., 2014).

Los registros de temperatura de la superficie del mar alrededor de las islas Canarias muestran una tendencia media al calentamiento de $0,28^{\circ} \mathrm{C}$ por década, para el periodo 1982-2013, pero con una gran variabilidad espacial. En el margen continental las tendencias van desde casi cero en la región de afloramiento (Sáhara occidental), a más de $0,5^{\circ} \mathrm{C}$ por década en la zona de hundimiento (entre Cabo Verde y cabo Blanco). Las observaciones hidrográficas en la región oceánica de la zona de transición costera durante el periodo 1997-2017 indican unas tendencias similares a las observadas por encima de la termoclina permanente
(200-600 dbar). Las observaciones hidrográficas obtenidas en aguas superficiales (50-150 dbar) de la región de afloramiento indican que durante los últimos 20 años ha habido una disminución de su temperatura y la salinidad, coherente con la intensificación de los vientos favorables al afloramiento asociada al calentamiento global. En términos de densidad, la tasa de calentamiento de las capas superiores se ha visto compensada por el aumento de salinidad, que ha sido de 0,02 por década. Las aguas intermedias y las de la capa superior de las profundas no muestran ninguna tendencia estadísticamente significativa. Por el contrario, las aguas más profundas (2600-3600 m) al norte de las islas Canarias presentan unas tasas de enfriamiento y disminución de salinidad de $0,01^{\circ} \mathrm{C}$ y $0,002^{\circ}$ por década, respectivamente (Vélez-Belchí et al., 2015).

\section{Nivel del mar}

Las observaciones del nivel del mar provienen de dos fuentes: los registros de mareógrafos y la altimetría por satélite. Durante la última década, ambos conjuntos de datos han mostrado cambios significativos que han permitido mejorar el conocimiento sobre la variabilidad del nivel del mar y sus tendencias.

Con respecto a la altimetría por satélite, la principal mejora proviene de la extensión de los registros, que en estos momentos ya cuentan con casi 25 años, junto con los esfuerzos para mejorar el procesado de los datos y correcciones geofísicas aplicadas. Ello ha permitido actualizar las tasas de aumento del nivel del mar, que habían sido evaluadas previamente en base a registros más cortos. En el mar Mediterráneo, por ejemplo, la altimetría por satélite muestra un incremento promedio del nivel del mar absoluto (geocéntrico) de 2,6 0,2 mm/año durante el periodo 1993-2015 (Marcos et al., 2016). En ubicaciones particulares se muestran tendencias lineales que oscilan entre -4 y $+6 \mathrm{~mm} / \mathrm{año}$, pero las tendencias positivas o negativas más extremas suelen estar más asociadas a la variabilidad de la circulación oceánica que a procesos persistentes a largo plazo. En el Atlántico nororiental, los valores de las tendencias son en su mayoría positivos (entre 1,0 y 4,5 mm/año (Pérez-Gómez et al., 2015; Ablain et al., 2017) y consistentes con los observados en el Mediterráneo. La estructura espacial de las tendencias muestra tanto la señal de la dinámica de mesoescala como la de gran escala.

En relación a los registros de los mareógrafos, ha habido una importante mejora en la estimación de los movimientos verticales (VLM) de la corteza terrestre, que en las ubicaciones de los mareógrafos, ha permitido separar las tendencias no climáticas de la contribución del océano. Así, restando la señal de VLM a los registros de los mareógrafos se ha obtenido una mayor coherencia espacial y, por lo tanto, una mejor comprensión de los procesos subyacentes a la variabilidad del nivel del mar en la costa (Wöppelmann y Marcos, 2012). Además, la extracción de los VLM es clave para poder comparar las tendencias del nivel del mar obtenidas mediante mareógrafos costeros con observaciones geocéntricas de altimetría. Con la digitalización y control de calidad de los registros en papel de mareógrafos antiguos y su intercomparación con medidas independientes 
de nivel del mar, se han podido recuperar largas series de datos y estimar tendencias a largo plazo del nivel del mar, como por ejemplo, el registro de Tenerife (Marcos et al., 2013). Durante la última década, también se han obtenido y mejorado las reconstrucciones regionales del campo de nivel del mar basadas en la combinación de altimetría y registros de mareógrafos, por ejemplo, en el Mediterráneo (Calafat y Jordà, 2011).

Durante la última década también ha habido un avance significativo en la comprensión de los procesos subyacentes a la variabilidad del nivel del mar. Por ejemplo, en lo que respecta al acoplamiento entre la variabilidad del nivel del mar en el Mediterráneo y en el Atlántico nororiental (Calafat et al., 2012). Estos autores han puesto en evidencia que, además de la respuesta barotrópica a la presión atmosférica local y el viento, las variaciones costeras del nivel del mar en el límite oriental del Atlántico Norte (las que fuerzan el nivel del mar Mediterráneo) también muestran una respuesta baroclínica forzada por la componente del viento paralela a la costa. Otro avance ha sido la formulación del papel de la salinidad en la variabilidad del nivel del mar (Jordà y Gomis, 2013), que ha sido crucial para corregir las estimaciones previas de tendencias del nivel del mar en el Mediterráneo. Estos autores han demostrado que en mares semicerrados, la salinidad no solo contribuye negativamente a la componente estérica del nivel del mar, sino que al mismo tiempo contribuye positivamente a la componente de masa, por lo que ambas contribuciones prácticamente se cancelan.

Por último, también ha habido avances en el campo de la modelización regional (véanse los dos artículos de Jordà et al. en esta misma publicación). La razón de ser de los modelos regionales es que el diagnóstico del nivel del mar mediante modelos climáticos globales (MCG) no es un problema en sí mismo, pero esos modelos globales tienen poca resolución espacial que no es suficiente para resolver procesos relevantes en mares marginales, como la FADP en el Mediterráneo. En estos dominios se requiere, por tanto, modelos climáticos regionales (MCR) anidados con los MCG; en cuyo caso el problema se traslada a la configuración de las condiciones de contorno del MCR, que aunque sean correctas desde el punto de vista hidrodinámico, impiden un diagnóstico correcto del nivel del mar en el dominio regional (Adloff et al., 2105). Este inconveniente ya ha sido reconocido (Slangen et al., 2017) y se espera que quedará corregido en la próxima generación de MCR.

\section{Conclusiones y observaciones finales}

Los resultados obtenidos confirman los impactos del calentamiento global en el océano a escala regional ibérica. Estos impactos a menudo se presentan como cambios repentinos o en forma de saltos, en lugar de observarse como tendencias uniformes. Dicho comportamiento es muy relevante, aunque a veces pase desapercibido, ya que puede aumentar la incertidumbre de las predicciones. En este artículo se resalta la importancia del seguimiento rutinario a largo plazo de las aguas marinas. Por desgracia, la mayor parte de la financiación dedicada a la investigación marina se basa en proyectos con un recorrido temporal limitado, normalmente entre 3 y 4 años. Sin embargo, también es cierto que muchas de las observaciones en las que se basa el presente informe provienen de actividades de seguimiento y vigilancia que, si bien comenzaron a través de determinados proyectos científicos, actualmente se siguen manteniendo por parte del IEO y otras instituciones como Puertos del Estado o universidades. Este es el caso del IEOOS (Tel et al., 2016) y de la estación de seguimiento fondeada en el umbral de Espartel, en el estrecho de Gibraltar. Otras estaciones de vigilancia comenzaron como iniciativas voluntarias de muy bajo coste, por ejemplo la red Hydrochanges o algunas de las estaciones costeras más antiguas (Aquarium de San Sebastián o la estación de L'Estartit). En todos los casos, la utilidad de estas observaciones para seguir la evolución del cambio climático (además de para el estudio de los recursos biológicos y su dependencia de las variables fisicoquímicas, que son aspectos cruciales de la implementación de la Estrategia Marina de la UE) está más que demostrada. En cualquier caso, una estrategia más coordinada, que tuviera en cuenta la capacidad global del sistema de observación para detectar señales de cambio climático (en la línea del estudio de Llasses et al., 2015) sería muy bienvenida.

\section{Referencias}

Ablain, M., Legeais, J. F., Prandi, P., Marcos, M., FenoglioMarc, L., Dieng, H. B., Benveniste, J., Cazenave, A., 2017: Satellite Altimetry-Based Sea Level at Global and Regional Scales. Surv. Geophys., 38, 7-31.

Adloff, F., Somot, S., Sevault, F., Jordà, G., Aznar, R., Déqué, M., Herrmann, M., Marcos, M., Dubois, C., Padorno, E., Álvarez-Fanjul, E., Gomis, D., 2015: Mediterranean Sea response to climate change in an ensemble of twenty first century scenarios. Clim. Dyn., 45, 2775-2802.

Borghini, M., Bryden, H., Schroeder, K., Sparnocchia, S. and Vetrano, A., 2014: The Mediterranean is becoming saltier. Ocean Sci., 10, 693-700.

Calafat, F. M., Jordà, G., 2011: A Mediterranean sea level reconstruction (1950-2008) with error budget estimates. Global and Planetary Change, 79 (1-2), 118-133.

Calafat, F. M., Chambers, D. P., Tsimplis, M. N., 2012: Mechanisms of decadal sea level variability in the eastern North Atlantic and the Mediterranean Sea. J. Geophys. Res. Oceans, 117, C09022.

CIESM, 2009: Dynamics of Mediterranean deep waters. CIESM Workshop Monographs, 38, 132 pp.

Durrieu de Madron, X., Houpert, L., Puig, P., Sánchez-Vidal, A., Testor, P., Bosse, A., Estournel, C., Somot, S., Bourrin, F., Bouin, M. N., Beauverger, M., Beguery, L., Calafat, A., Canals, M., Cassou, C., Coppola, L., Dausse, D., D’Ortenzio, F., Font, J., Heussner, S., Kunesch, S., Lefevre, D., Le Goff, H., Martín, J., Mortier, L., Palanques, A., Raimbault, P., 2013: Interaction of dense shelf water cascading and open-sea convection in the northwestern Mediterranean during winter 2012. Geophys. Res. Lett., 40, 1379-1385. 
Durrieu de Madron, X., Ramonden, S., Berline, L., Houpert, L., Bosse, A., Martini, S., Guidi, L., Conan, P., Curtil, C., Delsaut, N., Kunesch, S., Ghiglione, J. F., Marsaleix, P., Pujo-Pay, M., Severin, T., Testor, P., Tamburini, C. and the ANTARES collaboration, 2017: Deep sediment resuspension and thick nepheloid layer generation by open-ocean convection. J. Geophys. Res. Oceans, 122.

Font, J., Puig, P., Salat, J., Palanques, A., Emelianov, M., 2007: Sequence of hydrographic changes in the NW Mediterranean deep water due to the exceptional winter 2005. Sci. Mar., 72, 339-346.

García-Lafuente, J., Sánchez Román, A., Díaz del Río, G., Sannino, G., Sánchez-Garrido, J. C., 2007: Recent observations of seasonal variability of the Mediterranean outflow in the Strait of Gibraltar, J. Geophys. Res., 112, C10005.

Goikoetxea, N., Borja, A., Fontán, A., González, M., Valencia, V., 2009: Trends and anomalies in sea-surface temperature, observed over the last 60 years, within the southeastern Bay of Biscay. Contin. Shelf Res., 29, 1060-1069.

González, M., Fontán, A., Esnaola, G., Collins, M., 2013: Abrupt changes, multidecadal variability and long-term trends in sea surface temperature and sea level datasets within the southeastern Bay of Biscay. J. Mar. Syst., 109-110, S144-S152.

Häkkinen, S., Rhines, P. B. and Worthen, D. L., 2015: Heat content variability in the North Atlantic Ocean in ocean reanalyses, Geophys. Res. Lett., 42, 2901-2909.

Hernández-Guerra, A., Pelegrí, J. L., Fraile-Nuez, E., BenítezBarrios, V., Emelianov, M., Pérez-Hernández, M. D., VélezBelchí, P., 2014: Meridional overturning transports at $7.5^{\circ} \mathrm{N}$ and $24.5^{\circ} \mathrm{N}$ in the Atlantic Ocean during 1992-93 and 2010-11. Prog. in Oceanogr., 128, 98-114.

Larsen, K. M. H., González-Pola, C., Fratantoni, P., Beszczynska-Möller, A., and Hughes, S. L. (eds), 2016: ICES Report on Ocean Climate 2015. ICES Cooperative Research Report, 331.79 pp.

Jordà, G., Gomis, D., 2013: On the interpretation of the steric and mass components of sea level variability: The case of the Mediterranean basin. J. Geophys. Res. Oceans, 118.

Lacombe, H., Tchernia, P., Gamberoni, L., 1985: Variable bottom water in the Western Mediterranean basin. Prog. in Oceanogr., 14, 319-338.

Levitus, S., Antonov, J. I., Boyer, T. P., Baranova, K., García, H. E., Locarnini, R. A., Mishonov, A. V., Reagan, J. R., Seidov, D., Yarosh, E. S., Zwenget, M. M., 2012: World ocean heat content and thermosteric sea level change (0-2000 m), 1955-2010, Geophys. Res. Lett., 39, L10603.

Llasses, J., Jordà, G., Gomis, D., 2015: Skills of different hydrographic networks to capture changes in the Mediterranean Sea at climate scales. Climate Research, 63, 1-18.
López-Jurado, J. L., González-Pola, C., Vélez-Belchí, P., 2005: Observation of an abrupt disruption of the longterm warming trend at the Balearic Sea, western Mediterranean Sea, in summer 2005. Geophys. Res. Lett., 32, L24606.

Marcos, M., Puyol, B., Calafat, F. M., Wöppelmann, G., 2013: Sea level changes at Tenerife Island (NE Tropical Atlantic) since 1927. J. Geophys. Res. Oceans, 118, 112.

Marcos, M., Jordà, G., Le Cozannett, G., 2016: Sea level rise and its impacts on the Mediterranean, in AllEnvi (ed.) The Mediterranean region under climate change. A scientific update (coordinated by S. Thiébault and J-P Moatti). 22nd Conference of the Parties to the United Nations Framework Convention on Climate Change (COP22, Marrakech, 2016).

Naranjo, C., Sammartino, S., García-Lafuente, J., TaupierLetage, I., 2015: Mediterranean waters along and across the Strait of Gibraltar, characterization and zonal modification. Deep-Sea Res., Part 1, 105, 41-52.

Naranjo, C., García-Lafuente, J., Sammartino, S., SánchezGarrido, J. C., Sánchez-Leal, R., 2017: Thermohaline properties of the Mediterranean outflow over the past decade. Geophys. Res. Lett. (en revisión).

Pérez Gómez, B., Álvarez Fanjul, E., Marcos, M., Puyol, B., García, M. J., 2015: Sea level variability and trends in the Canary Current Large Marine Ecosystem. En: Valdés, L. and Déniz González, I. (eds.). Oceanographic and biological features in the Canary Current Large Marine Ecosystem. IOC UNESCO, Paris. IOC Technical Series, No. $115,309320$.

Prieto, E., González-Pola, C., Lavín, A., Holliday, N. P., 2015: Interannual variability of the northwestern Iberia deep ocean: Response to large-scale North Atlantic forcing. J. Geophys. Res. Oceans, 120.

Puig, P., Durrieu de Madron, X., Salat, J., Schroeder, K., Martín, J., Karageorgis, A. P., Palanques, A., Roullier, F., López-Jurado, J. L., Emelianov, M., Moutin, T., Houpert, L., 2013: Thick bottom nepheloid layers in the Western Mediterranean generated by deep dense shelf cascading. Prog. in Oceanogr., 111, 1-23.

Sammartino, S., García-Lafuente, J., Naranjo, C., SánchezGarrido, J. C., Sánchez-Leal, R., Sánchez-Román, A., 2015: Ten years of marine current measurements in Espartel Sill, Strait of Gibraltar. J. Geophys. Res., 120, 6309-6328.

Schroeder, K., Chiggiato, J., Bryden, H. L., Borghini, M., Ismail, S. B., 2016: Abrupt climate shift in the Western Mediterranean Sea. Sci. Rep., 6, 23009.

Slangen, A. B. A., Adloff, F., Jevrejeva, S., Leclercq, P. W., Marzeion, B., Wada, Y., Winkelmann, R., 2017: A Review of recent updates of sea-level projections at global and regional scales. Surv. Geophys., 38, 385-406. 
Somavilla, R., González-Pola, C., Rodríguez, C., Josey, S. A., Sánchez, R. F., and Lavín, A., 2009: Large changes in the hydrographic structure of the Bay of Biscay after the extreme mixing of winter 2005, J. Geophys. Res., 114, C01001.

Somavilla, R., González-Pola, C., Schauer, U. and Budéus, G., 2016: Mid-2000s North Atlantic shift: Heat budget and circulation changes. Geophys. Res. Lett., 43, 2059-2068.

Tel, E., Balbín, R., Cabanas, J. M., García, M. J., GarcíaMartínez, M. C., González-Pola, C., Lavín, A., López-Jurado, J. L., Rodríguez, C., Ruiz-Villareal, M., Sánchez-Leal, R. F., Vargas-Yáñez, M., Vélez-Belchi, P., 2016: IEOOS: The Spanish Institute of Oceanography Observing System. Ocean Sci. Discuss., 12, 345-353.

Sullivan, C., 2016: The North Atlantic Ocean's missing heat is found in its depths, Eos, 97, doi:10.1029/ 2016EO047009. Publicado el 3 de marzo de 2016.

Theocharis, A., Klein, B., Nittis, K., and Roether, W., 2002: Evolution and status of the Eastern Mediterranean Transient (1997-1999). J. Mar. Syst., 33-34, 91-116.

Ulses, C., Estournel, C., Puig, P., Durrieu de Madron, X., Marsaleix, P., 2008: Dense shelf water cascoading in the northwestern Mediterranean during the cold winter 2005. Quantification of the export through the Gulf of Lion and the Catalan margin. Geophys. Res. Lett., 35, L07610.

Vargas-Yáñez, M., Zunino, P., Schroeder, K., López-Jurado, J. L., Plaza, F., Serra, M., Castro, C., García-Martínez, M. C.,
Moya, F., Salat, J., 2012: Extreme Western Intermediate Water formation in Winter 2012. J. Mar. Syst., 105-108, 52-59.

Vargas-Yáñez, M., García-Martínez, M. C., Moya, F., Balbín, R., López-Jurado, J. L., Serra, M., Zunino, P., Pascual, J., Salat, J., 2017: Updating temperature and salinity mean values and trends in the Western Mediterranean: RADMED project. Prog. in Oceanogr., 157, 27-46.

Vélez-Belchí, P., Hernández-Guerra, A., Fraile-Nuez, E., Benítez-Barrios, V., 2010: Changes in Temperature and Salinity Tendencies of the Upper Subtropical North Atlantic Ocean at $24.5^{\circ}$ N. J. Phys. Oceanogr., 40, 2546-2555.

Vélez-Belchí, P., González-Carballo, M., Pérez-Hernández, M. D., Hernández-Guerra, A., 2015: Open ocean temperature and salinity trends in the Canary Current Large Marine Ecosystem. En: Valdés, L. and Déniz-González, I. (eds.). Oceanographic and biological features in the Canary Current Large Marine Ecosystem. IOC UNESCO, Paris. IOC Technical Series, 115, 299-308.

Wöppelmann, G., Marcos, M., 2012: Coastal sea level rise in southern Europe and the non-climate contribution of vertical land motion. J. Geophys. Res. Oceans, 117, C01007.

Zunino, P., Schroeder, K., Vargas-Yáñez, M., Gasparini, G. P., Coppola, L., García-Martínez, M. C., Moya, F., 2012: Effects of the Western Mediterranean Transition on the resident water masses: Pure warming, pure freshening and pure heaving. J. Mar. Syst., vol. 96-97, 15-23. 